J. clin. Path. (1948), 1, 99.

\title{
CHLORAL HYDRATE PLATES FOR THE INHIBITION OF SWARMING OF PROTEUS
}

\author{
BY \\ E. HARPER GILLESPIE \\ Public Health Laboratory Service, Leicester \\ (RECEIVED FOR PUBLICATION, JULY 17, 1947)
}

In routine bacteriology the swarming of Proteus on plate cultures often masks the identity or presence of other bacteria which may be present. Mixed cultures including Proteus are often obtained from open wounds or sinuses following abdominal or chest operations, so that unless some special selective medium is used it is usually impossible to identify or to isolate other bacteria from a culture containing Proteus. Frequently $\beta$-haemolytic streptococci are, missed in such cultures, and thus one often fails to identify an organism causing some infection which may have become secondarily infected with Proteus.

Numerous methods have been published for the inhibition of swarming of Proteus on culture plates, the s.mplest being the use of 6 per cent agar (Hayward and Miles, 1943). This high concentration of agar often leads to technical difficulties in preparation, and if the plates are not sufficiently dried, or if a very moist specimen is inoculated, Proteus is not necessarily prevented from swarming. Many chemicals have been incorporated in agar to make selective media, the most popular method being that of adding $1 / 5,000$ sodium azide (Snyder and Lichstein, 1940). Unfortunately, when sodium azide blood-agar plates are used the blood tends to haemolyse and the plates do not keep satisfactorily before use. Jones and Handley (1945) described a satisfactory highly selective medium for the isolation of Salmonellae from material contaminated with Proteus.

In 1931 Krämer and Koch reported the results of their experiments using 14 different types of media for the inhibition of swarming, and showed how the addition of chloral hydrate prevented the swarming of Proteus. In this country the practical use of chloral hydrate plates appears to be little known except in certain laboratories; this paper has therefore been written in order to bring it to the knowledge of everyone as being a practical method.

After certain preliminary experiments it has been found that chloral hydrate in a final concentration of $1 / 1,000$ in nutrient agar or blood agar inhibits the swarming of Proteus but allows good growth of Proteus and of most other bacteria. Experience with the method coincides with that of Krämer and Koch, who found that practically all bacteria produced typical colonies. One great advantage is that it allows good growth of $\beta$-haemolytic streptococci, and also permits the production of $\beta$ haemolysis, which thus enables one to detect and isolate single colonies from cultures heavily infected with Proteus. It is an advantage to incubate the plates for a full 24 hours, although after 18 hours the $\beta$ haemolysis of haemolytic streptococci can usually be detected with ease.

On 1/1,000 chloral hydrate plates colonies of Proteus appear as discrete colonies from 1 to $2 \mathrm{~mm}$. in diameter, so that if necessary one can pick off single colonies of Proteus and also single pure colonies of other bacteria from mixed cultures. This is an advantage over media which inhibit the growth of Proteus, as on them colonies of other bacteria need not necessarily be pure, as the Proteus bacilli just fail to grow but lie dormant and live on the plate. Weaker concentrations, such as $1 / 1,500$, allow the colonies of Proteus to become large and semi-confluent, making it difficult to isolate other types of colonies present. At $1 / 2,000$ slight spreading occurs.

Stronger concentrations inhibit the growths of Proteus and of other bacteria, but also inhibit the production of $\beta$ haemolysis on blood-agar plates. by haemolytic streptococci. 


\section{Preparation of Plates}

The method of preparation of chloral hydrate plates is simple. A stock 10 per cent solution of chloral hydrate is made in distilled water and an appropriate amount added to the medium to give a final strength of $1 / 1,000$. It is an advantage to make several plates at one time, adding the chloral hydrate to the bulk base at the time of pouring the plates, otherwise a very small volume must be added to the medium for each plate. It is not necessary to sterilize the stock solution, as it is apparently self-sterilizing at this strength, but it is an obvious advantage to exclude bacteria, especially spore-bearers, by using sterile distilled water. If preferred, the stock solution may be autoclaved without interfering with its inhibitory properties. Once the plates are poured they should be dried in the usual way, but not overdried.

The chloral hydrate blood-agar plates may be stored in the refrigerator for a week before use without the occurrence of haemolysis. Any stored plate should be dried in the incubator for about half an hour before inoculation.

Krämer and Koch report having found occasional batches of blood-agar plates giving atypical growths, but over the last three years no very unusual growth has been found as long as the cor- rect percentage is accurately added to the media. $\Rightarrow$ Slight increase in the concentration due to the inaccurate addition of too small a volume of stock solution will lead to irregular results.

\section{Conclusions}

1. Chloral hydrate in a final concentration of $1 / 1,000$ in nutrient agar or blood-agar plates inhibits the swarming of Proteus.

2. The chloral hydrate in the strength recommended permits the growth of Proteus as single? colonies 1 to $2 \mathrm{~mm}$. in diameter.

3. Chloral hydrate in this strength allows the production of $\beta$ haemolysis on blood-agar plates by haemolytic streptococci.

4. Chloral hydrate plates permit the identifica- $\vec{T}$ tion and isolation in pure culture of bacteria from $\frac{D}{O}$ mixed cultures contaminated with Proteus.

\section{REFERENCES}

Hayward, N. J., and Miles, A. A. (1943). Lancet, 2, 116. Jones, R. E., and Handley, W. R. C. (1945). Mon. Bull. Min. Hlth. publ. Hith. Lab. Serv., London, 4, 107.

Krämer, E., and Koch, F. E. (1931). Zhl. Bakt. (orig.), 120, 452. Snyder, M. L., and Lichstein, H. C. (1940). J. infect. Dis., 67, 113 